\title{
Visualization of Bioinformatics Data with Dash Bio
}

\author{
Shammamah Hossain ${ }^{\ddagger *}$
}

Abstract-Plotly's Dash is a library that empowers data scientists to create interactive web applications declaratively in Python. Dash Bio is a bioinformaticsoriented suite of components that are compatible with Dash. Visualizations of data that are often found in the field of bioinformatics can now be integrated into Dash applications. We present the Dash Bio suite of components and parts of an auxiliary library that contains tools that parse files from common bioinformatics databases.

Index Terms—visualization, bioinformatics, sequence analysis, Dash

\section{Introduction}

The emergent field of bioinformatics is an amalgamation of computer science, statistics, and biology; it has proven itself revolutionary in biomedical research. As scientific techniques in areas such as genomics and proteomics improve, experimentalists in bioinformatics may find themselves needing to interpret large volumes of data. In order to use this data to efficiently provide meaningful solutions to biological problems, it is important to have robust data visualization tools.

Many bioinformaticians have already created analysis and visualization tools with Dash and plotly.py, but only through significant workarounds and modifications made to preexisting graph types. We present an interface to create single-line declarations of charts for complex datasets such as hierarchical clustering and multiple sequence alignment. In addition, we introduce several new chart types, three-dimensional and interactive molecule visualization tools, and components that are specifically related to genomic and proteomic sequences. In a separate library, we present a set of simple parsing scripts that handle some of the most common file types found in bioinformatics-related databases.

This paper outlines the contents of the Dash Bio package. With this package, we hope to impart the powerful data-visualization tools and flexibility of Dash to the flourishing bioinformatics community.

\section{Dash}

Plotly's dash library provides a declarative Python interface for developing full-stack web applications ("Dash apps"). [Dash] In addition to the main dash library, the dash-html-components and dash-core-components packages comprise the building blocks of a Dash app. dash-html-components provides an interface for building

* Corresponding author: shammamah@plot.ly

† Plotly, Inc., 118 - 5555 Avenue de Gaspe, Montreal QC H2T $2 A 3$

Copyright $\odot 2019$ Shammamah Hossain. This is an open-access article distributed under the terms of the Creative Commons Attribution License, which permits unrestricted use, distribution, and reproduction in any medium, provided the original author and source are credited.

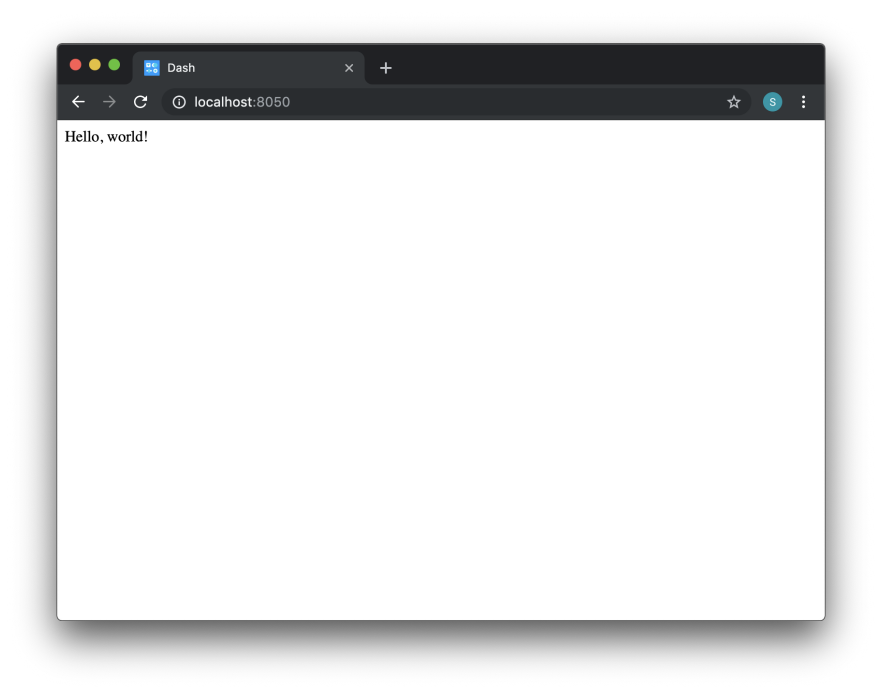

Fig. 1: A simple Dash application.

the layout of a Dash application that mimics the process of building the layout of a website; dash-core-components is a suite of common tools used for interactions with a Dash app (e.g., dropdowns, text inputs, and sliders) and includes a dcc.Graph component for interactive graphs made with plotly.py.

A minimal Dash application that comprises a string on a webpage can be produced with the following code.

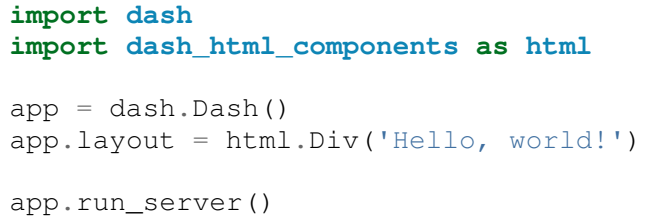

Upon running the above code, a local host address is specified in the console. Visiting this address in the browser yields a simple webpage that contains the text "Hello, world!" (see Fig. 1).

Interactivity is implemented with callbacks. These allow for reading the values of inputs in the Dash app (e.g., text inputs, dropdowns, and sliders), which can subsequently be used to compute the value of one or more "outputs", i.e., properties of other components in the app. The function that computes the outputs is wrapped in a decorator that specifies the aforementioned inputs and outputs; together, they form a callback. The callback is triggered whenever one of the specified inputs changes in value.

For instance, the dash_core_components. Input () component controls the children property of a dash_html_components.Div() component in the following code. 


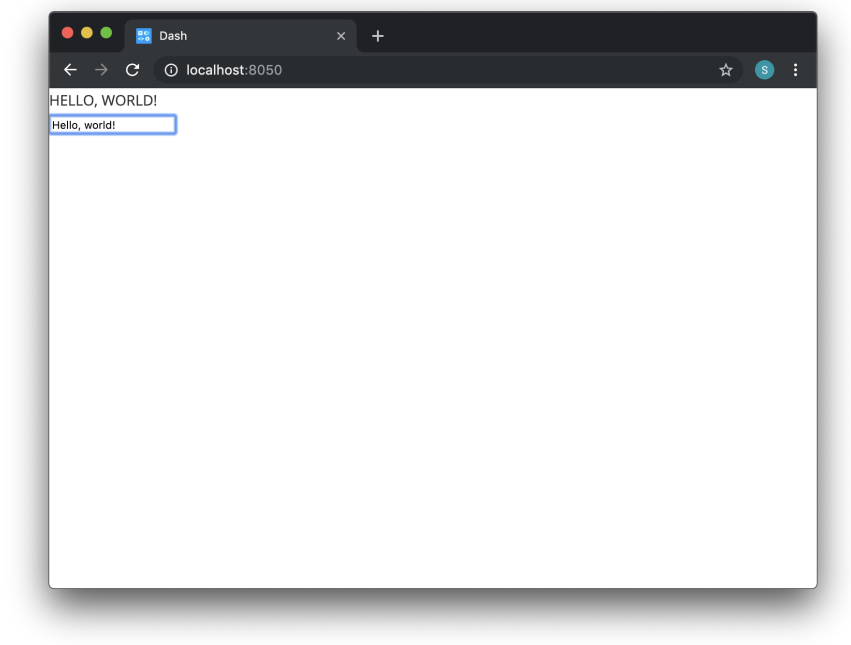

Fig. 2: A simple Dash application that showcases interactivity. Text that is entered into the input component is converted to uppercase and displayed in the app.

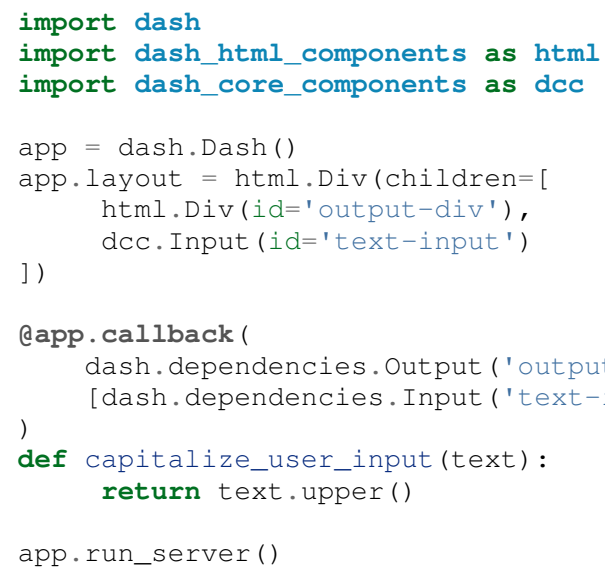

The output of the code is shown in Fig. 2.

\section{React.js and Python}

Some of the components in the Dash Bio package are wrappers around pre-existing JavaScript or React libraries. The development process for JavaScript-based components is fairly straightforward; the only thing that needs to be added in many cases is an interface for Dash to access the state of the component and read or write to its properties. This provides an avenue for interactions with the components from within a Dash app.

The package also contains three Python-based components: Clustergram, ManhattanPlot, and VolcanoPlot. Unlike the JavaScript-based components, the Python-based components are essentially functions that return JSON data that is in the format of the figure argument for a dash_core_components.Graph component.

\section{Dash Bio Components}

Dash Bio components fall into one of three categories.

- Custom chart types: Specialized chart types that allow for intuitive visualizations of complex data. This category includes Circos, Clustergram, Ideogram, ManhattanPlot, NeedlePlot, and VolcanoPlot.
- Three-dimensional visualization tools: Structural diagrams of biomolecules that support a wide variety of user interactions and specifications. This category includes Molecule3dViewer and Speck.

- Sequence analysis tools: Interactive and searchable genomic and proteomic sequences, with additional features such as multiple sequence alignment. This category include AlignmentChart, OncoPrint, and SequenceViewer.

The documentation for all of the Dash Bio components, including example code, can be found at https://dash.plot.ly/dash-bio.

\section{Circos}

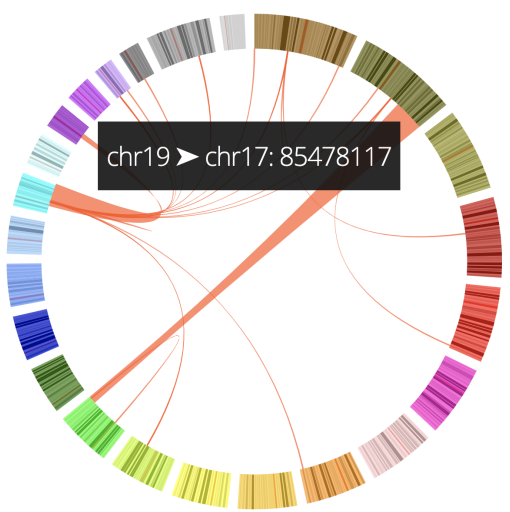

Fig. 3: A simple Dash Bio Circos component with chords connecting pairs of data points. Data taken from [Ghr] and converted to JSON in the Circos JS repository [Circos].

Circos is a circular graph. It can be used to highlight relationships between, for example, different genes by drawing chords that connect the two (see Fig. 3).

The Dash Bio Circos component is a wrapper of the CircosJs [Circos] library, which supports additional graph types like heatmaps, scatter plots, histograms, and stacked charts. Input data to Circos take the form of a dictionary, and are supplied to the layout parameter of the component. Additional data, such as a list of chords, are specified in the tracks parameter. Multiple tracks can be plotted on the same Circos graph. Hover data and click data on all Circos graph types are captured and are available to Dash apps.

\section{Clustergram}

A clustergram is a combination heatmap-dendrogram that is commonly used in gene expression data. The hierarchical clustering that is represented by the dendrograms can be used to identify groups of genes with related expression levels.

The Dash Bio Clustergram component is a Python-based component that uses plotly.py to generate a figure. It takes as input a two-dimensional numpy array of floating-point values. Imputation of missing data and computation of hierarchical clustering both occur within the component itself. Clusters that meet or exceed a user-defined threshold of similarity comprise single traces in the corresponding dendrogram, and can be highlighted with annotations (see Fig. 4).

The user can specify additional parameters to customize the metrics and methods used to compute parts of the clustering, such as the pairwise distance between observations and the linkage 


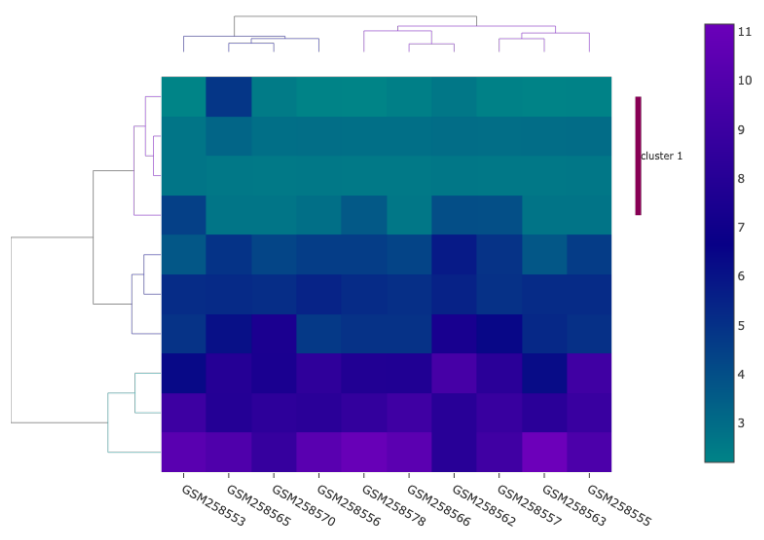

Fig. 4: A Dash Bio clustergram component displaying hierarchical clustering of gene expression data from two lung cancer subtypes. A cluster from the row dendrogram (displayed to the left of the heatmap) is annotated. Data taken from [KR09].

matrix. Hover data and click data are accessible from within the Dash app for the heatmap and both dendrograms that are shown in Fig. 4.

\section{Ideogram}

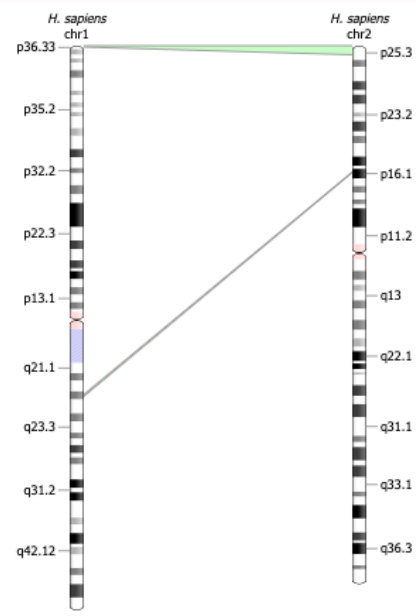

Fig. 5: A Dash Bio ideogram component demonstrating the homology feature with two human chromosomes. Data taken from the ideogram. js repository [Ideo].

An ideogram is a schematic representation of genomic data. Chromosomes are represented as strands, and the locations of specific genes are denoted by bands on the chromosomes.

The Dash Bio Ideogram component is built on top of the ideogram.js library [Ideo], and includes features like annotations, histograms, and homology (see Fig. 5). Annotations can be made to different segments of each chromosome and displayed in the form of bands, and relationships between different chromosomes can be highlighted by using the homology feature to connect a region on one chromosome to a region on another (see Fig. 5). Upon hovering over an annotated part of the chromosome, the annotation data is readable from within a Dash app. Additionally, information from the the "brush" feature, which allows the user to highlight a subset of the chromosome, is accessible from within the Dash application. This information includes the starting position and ending position of the brush, as well as the length (in base pairs) of the selection made with the brush.

\section{Manhattan Plot}

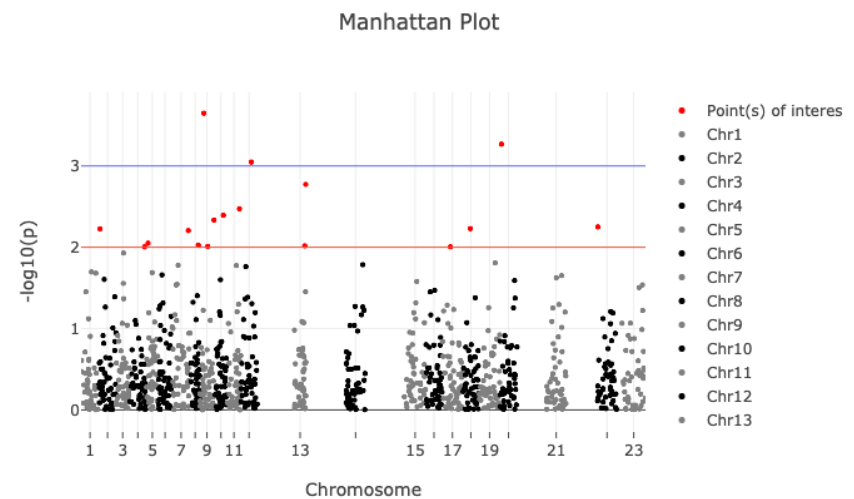

Fig. 6: A Dash Bio ManhattanPlot component. The threshold level is denoted by the red line; all points of interest are colored red. The purple line is the suggestive line. Data taken from the manhat tan Iy repository [Man].

A Manhattan plot is a plot commonly used in genome-wide association studies; it can highlight specific nucleotides that, when changed to a different nucleotide, are associated with certain genetic conditions.

The Dash Bio ManhattanPlot component is built with plotly.py. Input data take the form of a pandas dataframe. The two lines on the plot (see Fig. 6) represent, respectively, the threshold level and the suggestive line. ${ }^{1}$ The $y$-values of these lines can be controlled by the user. Hover data and click data are accessible from within the Dash app.

\section{Needle Plot}

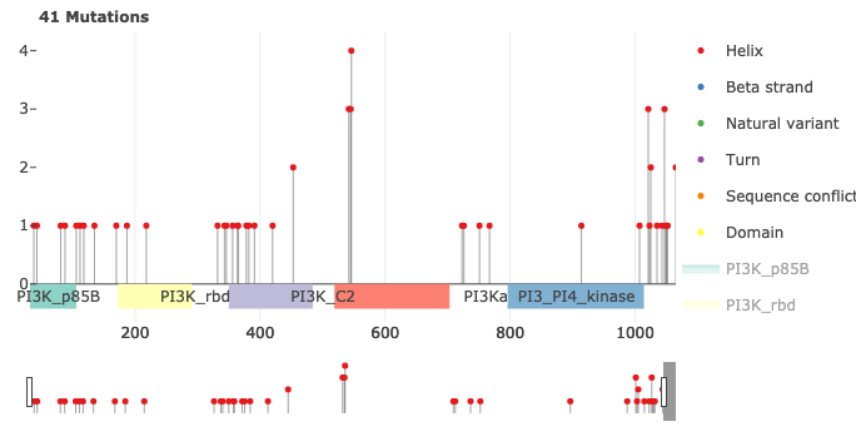

Fig. 7: A Dash Bio NeedlePlot component that shows the properties of mutations in a genomic strand. Data taken from the muts-needle-plot repository [Muts].

A needle plot is a bar plot in which each bar has been replaced with a marker at the top and a line from the $\mathrm{x}$-axis to

1. Information about the meaning of these two lines can be found in [ER15] 
the aforementioned marker. Its primary use-case is visualization of dense datasets that would appear too crowded to be interpreted effectively when represented with a bar plot. In bioinformatics, a needle plot may be used to annotate the positions on a genome at which genetic mutations happen (see Fig. 7).

The Dash Bio NeedlePlot component was built using plotly.js. It receives input data as a dictionary. Different colors and marker styles can be used to distinguish different types of mutations, and the domains of specific genes can be demarcated on the plot.

Volcano Plot

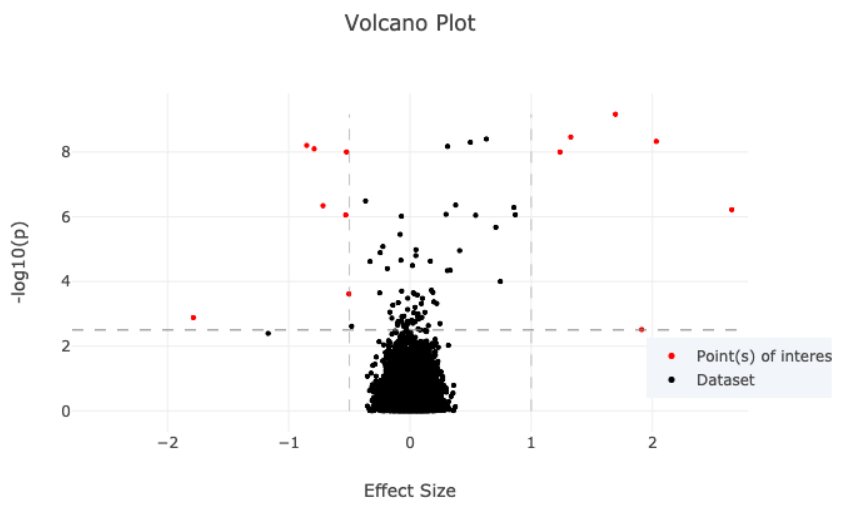

Fig. 8: A Dash Bio VolcanoPlot component. Points of interest are colored in red, and the effect size and statistical significance thresholds are represented by dashed lines. Data taken from the manhat tan Iy repository [Man].

A volcano plot is a plot used to concurrently display the statistical significance and a defined "effect size" (e.g., the fold change $^{2}$ ) of a dataset. This type of plot is incredibly useful when visualizing a large number of data points that represent replicate data; it facilitates identification of data that simultaneously have statistical significance and a large effect.

The Dash Bio VolcanoPlot component was built using plotly.py. It takes a pandas dataframe as input data. Lines that represent the threshold for effect size (both positive and negative) and a threshold for statistical significance can be defined by the user (see Fig. 8). Hover data and click data are accessible from within the Dash app.

\section{Molecule 3D Viewer}

The Dash Bio Molecule3dViewer component was built on top of the molecule-3d-for-react [Mol3D] library. Its purpose is to display molecular structures. These types of visualizations can show the shapes of proteins and provide insight into the way that they bind to other molecules. This renders them invaluable when communicating the mechanics of biomolecular processes.

Molecule3dViewer receives input data as a dictionary which specifies the layout and style of each atom in the molecule. It can render molecules in a variety of styles, such as ribbon diagrams, and allows for mouse-click selection of specific atoms or residues (see Fig. 9) that can be read from or written to within a Dash app.

\section{Speck}

The Dash Bio Speck component is a WebGL-based 3D renderer that is built on top of Speck [Speck]. It uses techniques like

2. This refers to the ratio of a measurement to its preceding measurement.

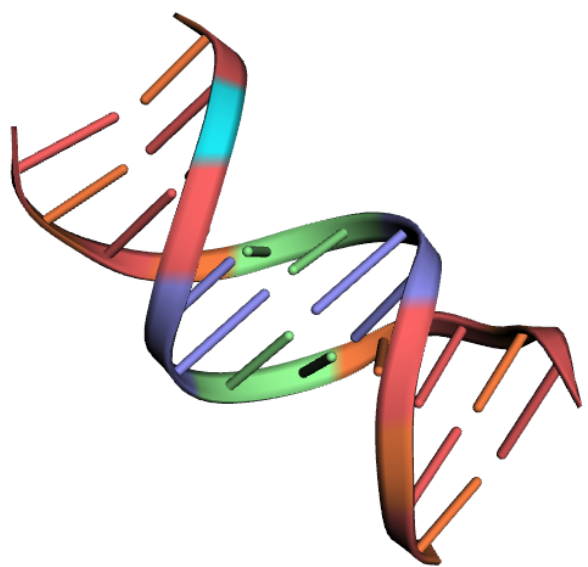

Fig. 9: A Dash Bio Molecule3DViewer component displaying the ribbon structure of a section of DNA. A selected residue is highlighted in cyan. Structural data taken from the Protein Data Bank [1bna].

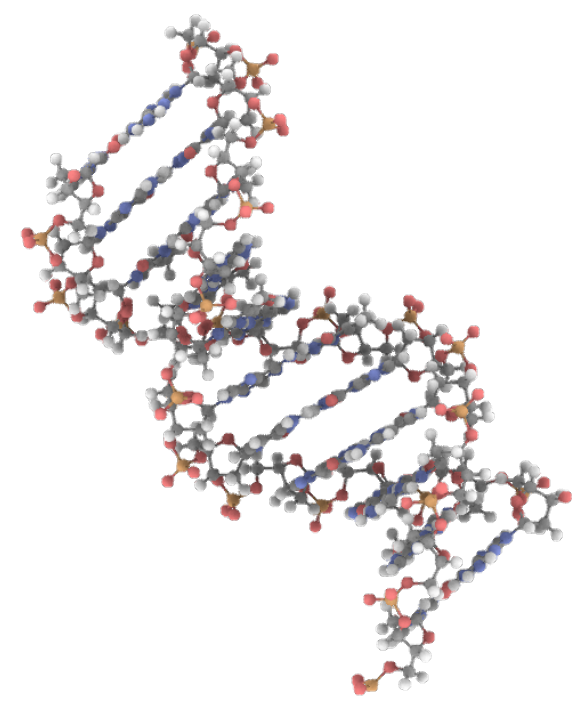

Fig. 10: A Dash Bio Speck component displaying the atomic structure of a strand of DNA in a ball-and-stick representation. Ambient occlusion is used to provide realistic shading on the atoms. Structural data taken from the Speck repository [Speck].

ambient occlusion and outlines to provide a rich view of molecular structures (see Fig. ).

The Dash Bio Speck component receives input data as a dictionary that contains, for each atom, the atomic symbol and the position in space (given as $\mathrm{x}, \mathrm{y}$, and $\mathrm{z}$ coordinates). Parameters related to the rendering of the molecule, such as the atom sizes, levels of ambient occlusion, and outlines, can optionally be specified in another dictionary supplied as an argument.

\section{Alignment Chart}

An alignment chart is a tool for viewing multiple sequence alignment. Multiple related sequences of nucleotides or amino acids (e.g., the amino acid sequences of proteins from different 


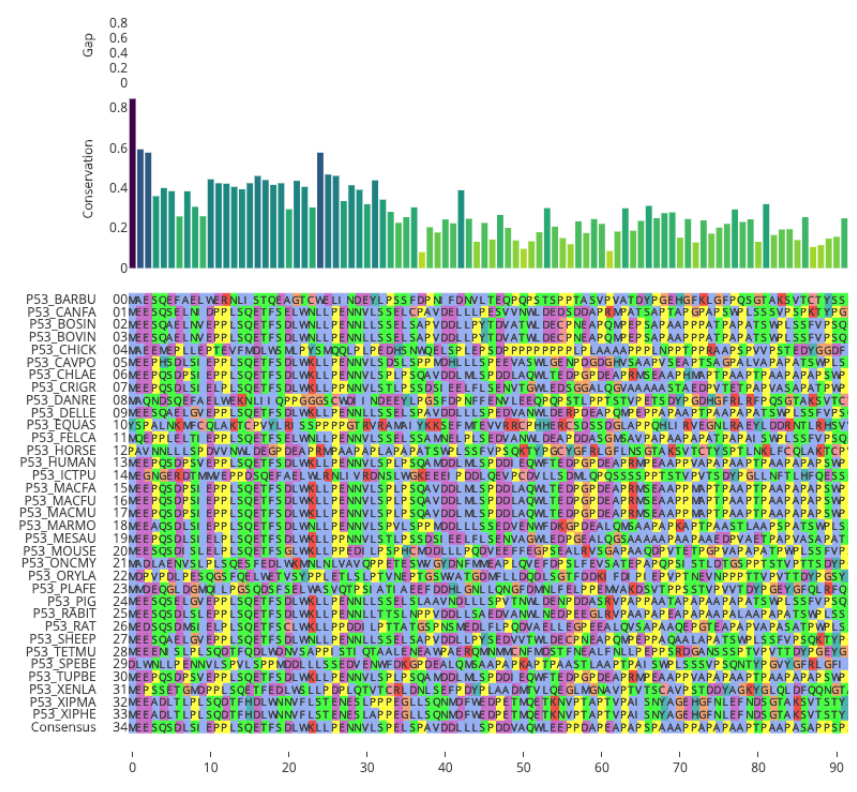

Fig. 11: A Dash Bio AlignmentChart component displaying the P53 protein's amino acid sequences from different organisms. A conservation barplot is displayed on top, and the bottom row of the heatmap contains the consensus sequence. Data taken from UniProt [UniP].

organisms that appear to serve the same function) are displayed in the chart to show their similarities.

The Dash Bio AlignmentChart component is built on top of react-alignment-viewer [Align]. It takes a FASTA file as input and computes the alignment. It can optionally display a barplot that represents the level of conservation of a particular amino acid or nucleotide across each sequence defined in the input file (see Fig. 11). Hover data and click data are accessible from within the Dash app.

\section{Onco Print}

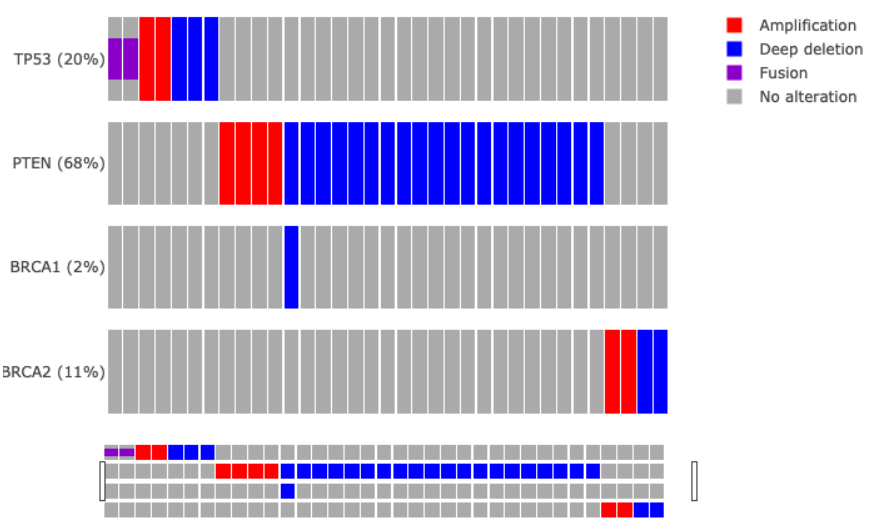

Fig. 12: A Dash Bio OncoPrint component that shows mutation events for the genomic sequences that encode different proteins. Data taken from cBioPortal [cBio], [cBio2].

An OncoPrint graph is a type of heatmap that facilitates the visualization of multiple genomic alteration events (see Fig. 12).

The Dash Bio OncoPrint component is built on top of react-oncoprint [Onco]. Input data for the component takes the form of a list of dictionaries that each define a sample, gene, alteration, and mutation type. Hover data and click data are accessible from within the Dash app.

\section{Sequence Viewer}

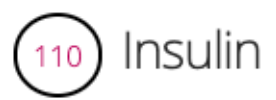

Search in sequence.. (Regex supported)

1 MALWMRLLPL LALLALWGPD PAAAFVNOHIL CGSHLVEALY

41 LVCGERGF TPKTRREAED LQVGQVELGG GPGAGSLPL

81 ALEGSLQKRG IVEQCCTSIC SLYQLENYC

Fig. 13: A Dash Bio SequenceViewer component that is showing the amino acid sequence for insulin. A coverage has been applied to the sequence to emphasize subsequences of amino acids that form certain structures, like alpha helices or beta sheets. Data taken from NeXtProt $[n X P]$.

The Dash Bio SequenceViewer component is a simple tool that allows for annotating genomic or proteomic sequences. It is based on the react-sequence-viewer library [SeqV].

It includes a search function that allows the user to search the sequence using regular expressions. In addition, the sequence can be annotated using a selection defined by a starting point, an end point, and a color, or a coverage that can encode additional information that is revealed once a subsequence is clicked. The selection and coverage are available for reading from and writing to in the Dash app, and the mouse selection and search results are also accessible.

\section{File Parsers}

The dash-bio-utils package was developed in tandem with the dash-bio package. It contains parsers for common filetypes used in bioinformatics analyses. The parsers in the package translate the data encoded in those files to inputs that are compatible with Dash Bio components.

\section{FASTA data}

FASTA files are commonly used to represent one or more genomic or proteomic sequences. Each sequence may be preceded by a line starting with the $>$ character which contains information about the sequence, such as the name of the gene or organism; this is the description of the sequence. Sections of the description are separated with pipes $(\mid)$.

The protein_reader file in the dash-bio-utils package accepts a file path to, or a string representation of, a FASTA file, and returns a dictionary that contains the sequence and any metadata that are specified in the file. SeqIO from the Biopython [BioP] package was used to extract all of the sequences from the file into a list of dictionaries, each of which contained the sequence description and the sequence itself, both in string format.

Different databases (e.g., neXtProt, GenBank, and SWISSPROT) encode the sequence description metadata in different ways. The database from which a FASTA file is retrieved is 
specified in the first line. In the protein_reader file, the code for the database is translated into the information that is encoded in the first line for that particular database. [NCBI]

From there, string splitting (or, if necessary, regex) is used on the description line of the file to generate a dictionary of the sequence metadata.

This parser enables quick access to all of the information contained in a FASTA file, which in turn can make the information more human-readable. This is a feature that supplements the easeof-use of the dash-bio package.

For instance, in the code snippet below, the parser is used on a string with the contents of a FASTA file for the albumin protein $[\mathrm{nXP}]$ :

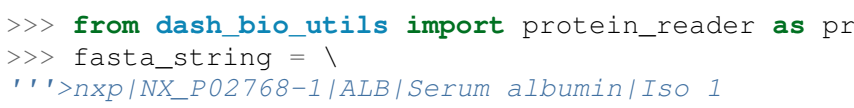

MKWVTF I SLLFLFS SAYSRGVFRRDAHKSEVAHRFKDLGEENFKALVLIAF $A Q Y L Q Q C P F E D H V K L V N E V T E F A K T C V A D E S A E N C D K S L H T L F G D K L C T V A$ TLRETYGEMADCCAKQEPERNECFLQHKDDNPNLPRLVRPEVDVMCTAFHD NEETFLKKYLYEIARRHPYFYAPELLFFAKRYKAAFTECCQAADKAACLLP KLDELRDEGKAS SAKQRLKCASLQKFGERAFKAWAVARLSQRFPKAEFAEV SKLVTDLTKVHTECCHGD LLECADDRADLAKY I CENQD S I S SKLKECCEKP LLEKSHCIAEVENDEMPADLP SLAADFVESKDVCKNYAEAKDVFLGMFLYE YARRHPDYSVVLLLRLAKTYETTLEKCCAAADP HECYAKVFDEFKP LVEEP QNLIKQNCELFEQLGEYKFQNALLVRYTKKVPQVSTPTLVEVSRNLGKVGS KCCKHPEAKRMP CAEDYLSVVLNQLCVLHEKTPVSDRVTKCCTESLVNRRP CFSALEVDETYVPKEFNAETFTFHAD ICT LSEKERQIKKQTALVELVKHKP KATKEQLKAVMDDFAAFVEKCCKADDKETCFAEEGKKLVAASQAALGL ' ' '

$>>$ albumin = pr.read_fastal

…

...) $[0]$

$>>$ albumin['description']

\{'identifier': 'NX_P02768-1',

'gene name': 'ALB',

'protein name': 'Serum albumin',

'isoform name': 'Iso 1'\}

$\gg>$ albumin ['sequence'] [: 10$]$

'MKWVTFISLL'

\section{Gene Expression Data}

Gene expression data take the form of two-dimensional arrays that measure expression levels for sets of genes under varying conditions.

A common format that is used to represent gene expression data is the SOFT format. These files can be found in large databases such as the Gene Expression Omnibus (GEO), [GEO] which contains gene expression data from thousands of experiments. SOFT files contain the expression data, as well as descriptive information pertaining to the specific genes and conditions that are in the dataset.

The gene_expression_reader file in the dash-bio-utils package accepts a path to, or a string representation of, a SOFT file or TSV file containing gene expression data. It can parse the contents of SOFT and TSV files, and return the numerical data and metadata that they contain. In addition, selection of a subset of the data (given by lists of selected rows and selected columns supplied to the parser) can be returned.

The GEOparse package [GEOP] was used to extract the numeric gene expression data to a pandas dataframe, in addition to the metadata, in SOFT files:

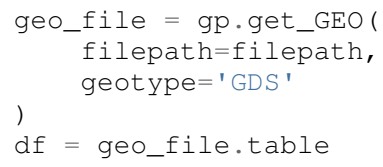

pandas was used to do the same with TSV files:

$\mathrm{df}=$ pd.read_csv (

filepath, $\operatorname{sep}=$ ' $\backslash t$ '

)

Both file parsers by default return a tuple comprising the file metadata, all of the row names, and all of the column names.

If the parameter return_filtered_data is set to True, the parameters rows and columns (lists that contain the names of, respectively, the selected rows and selected columns) must be specified. The dataframe $\mathrm{df}$ is then filtered according to these selections, and a two-dimensional numpy array containing the filtered data is returned.

In the case of SOFT files, there is additional information about subsets of the dataset (e.g., the expression data that are recorded with and without inducing a particular gene). This information becomes another element in the tuple.

In the code snippet below, the parser is used to extract information from a dataset related to the miR-221 RNA molecule [miR]:

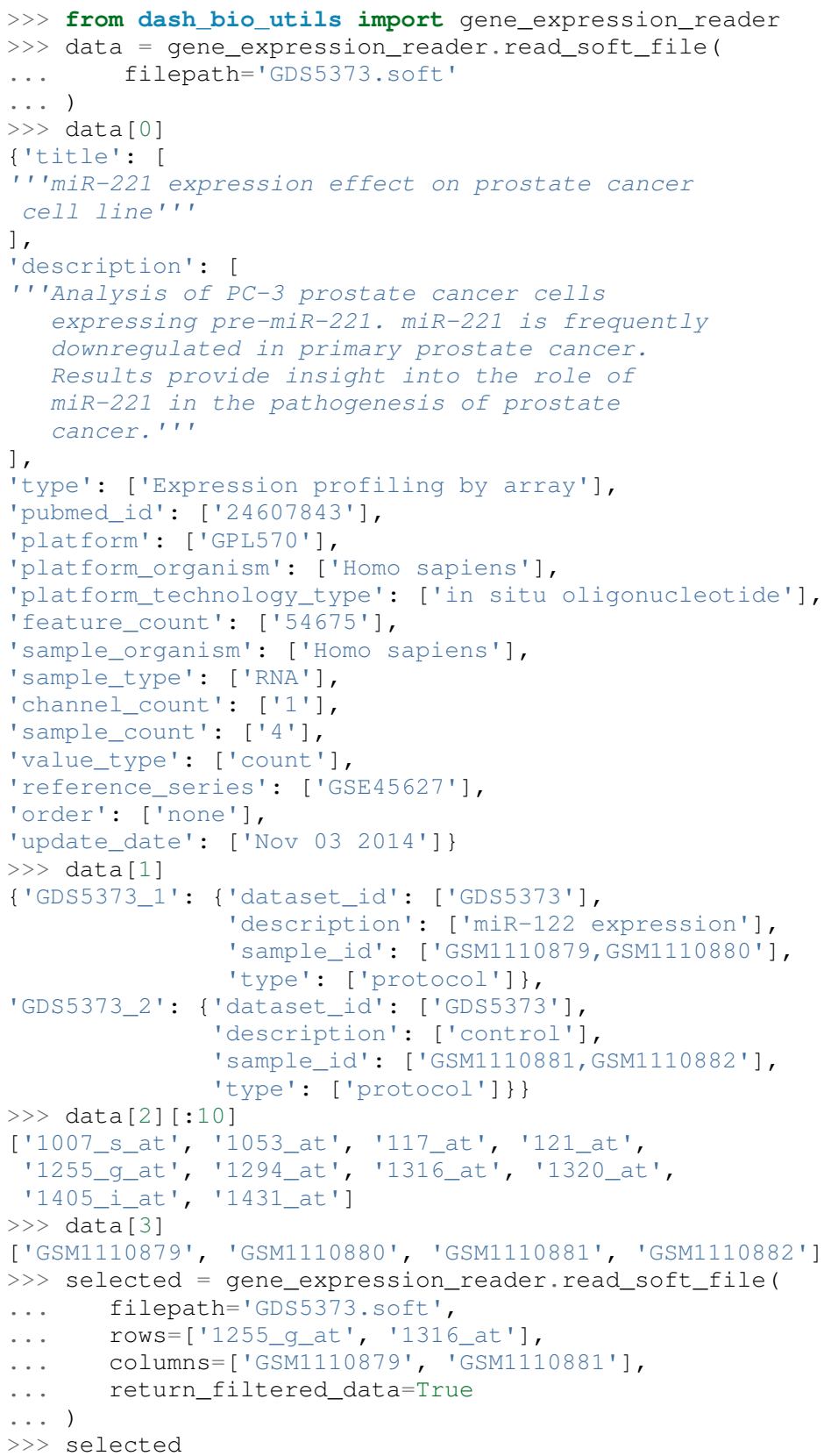


array ([ [22.7604, 23.0321], $[21.416,21.0107]])$

\section{Molecule Structural Data}

The Protein Data Bank (PDB) [PDB] is a database of files that describe macromolecular structural data. All of the files on PDB are in the PDB format.

In the dash_bio_utils package, the create_data function in pdb_parser generates a JSON string from the contents of a specified PDB file. This string contains information about the atoms and the bonds in the molecular structure.

The PDB format is standardized; properties of each atom such as its position in space and the chain and residue to which it belongs are found within specific column indices for each row. $[\mathrm{PdbF}] \mathrm{pdb} \_$parser uses this information to parse each line, and creates a list of dictionaries, each of which contains information about the aforementioned properties for each atom in the PDB file.

The parmed library [Par] was used to read the bond information from the PDB file. Using the bond information from parmed, a list of dictionaries is created; each dictionary contains the indices of the pair of atoms that form a bond.

In the code snippet below, this parser is used to extract data from a PDB file that contains structural information for a small section of DNA: [1bna]

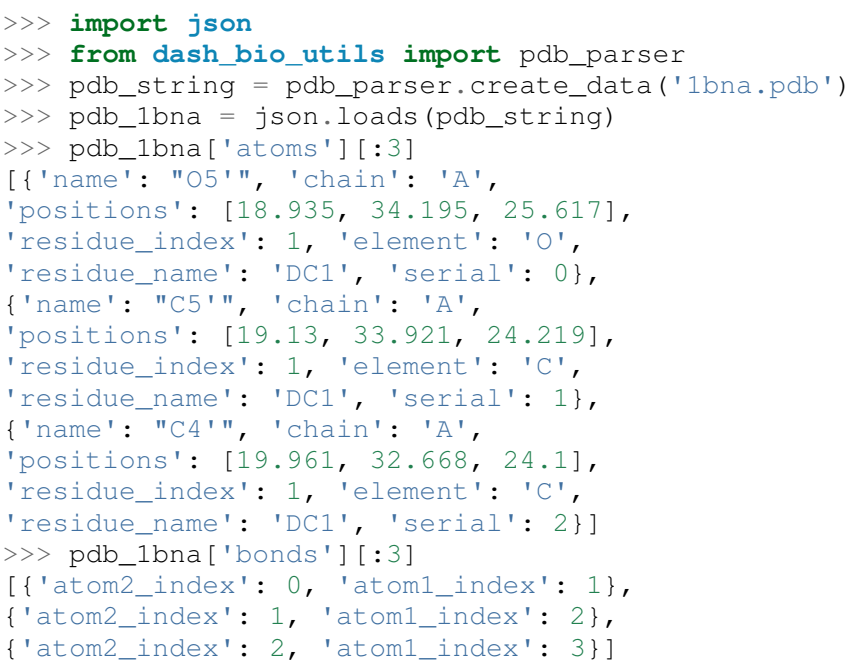

\section{Comparisons with Existing Tools}

\section{GenomeDiagram}

The GenomeDiagram package [Geno] provides a way to visualize comparative genomics data in a circular format (see Fig. 14); supported chart types include line charts, bar graphs, and heatmaps.

GenomeDiagram can additionally export high-quality vector diagrams of the charts that are generated, which can in turn be used in research papers. It can be used in conjunction with the BioPython module to interface with GenBank.

GenomeDiagram shares many similarities with the Circos component; both are circular representations of genomic data, and both support multiple "tracks", or traces, of multiple chart types. The key difference between the two, and the advantage of Dash Circos, is flexibility and interactivity; Dash Circos supports click and hover interactions, and GenomeDiagram does not.

Furthermore, Dash Circos can be interactively modified with respect to the data that are displayed, as well as the appearance of the graph itself. This allows for the implementation of many useful

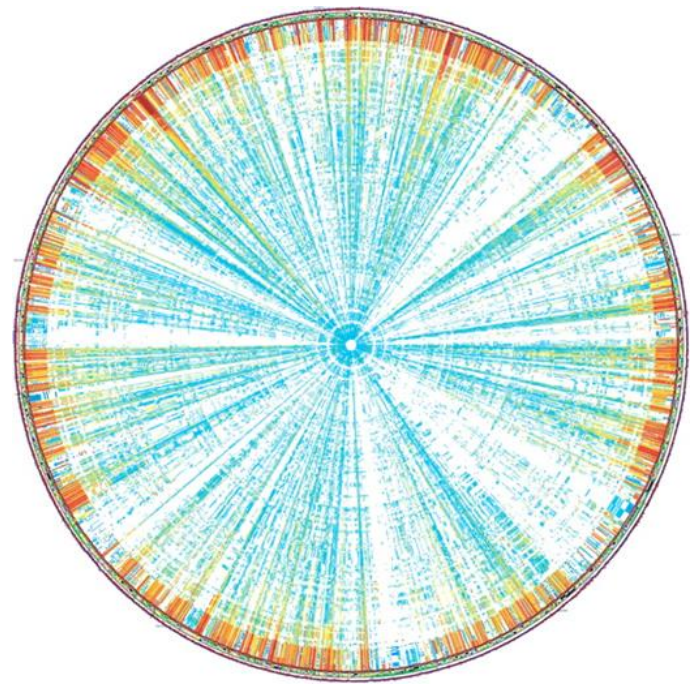

Fig. 14: An example of a circular diagram that can be generated with GenomeDiagram. Source: [Geno]

$\begin{array}{llllll}\text { Seq 1 } & \text { C } & \text { C } & \text { T } & \text { G } & \text { T } \\ \text { Seq 2 } & \text { C } & \text { C } & \text { T } & \text { G } & \text { T } \\ \text { Seq 3 } & \text { C } & \text { C } & \text { T } & \text { G } & \text { T } \\ \text { Seq 4 } & \text { C } & \text { C } & \text { T } & \text { G } & \text { T } \\ \text { Seq 5 } & \text { C } & \text { C } & \text { T } & \text { G } & \text { T } \\ \text { Seq 6 } & \text { C } & \text { C } & \text { T } & \text { G } & \text { T } \\ \text { Seq 7 } & \text { C } & \text { C } & \text { T } & \text { G } & \text { T } \\ \text { Seq 8 } & \text { C } & \text { C } & \text { T } & \text { G } & \text { T } \\ \text { Seq 9 } & \text { C } & \text { C } & \text { T } & \text { G } & \text { T }\end{array}$

Fig. 15: Part of a multiple sequence alignment displayed as a Plotly heatmap. Source: [JCViz]

functions, such as cross-filtering. Instead of needing to re-create the image every time a change is made, updates to the Circos component are reflected immediately within a Dash app.

\section{Plotly.py}

Plotly's Python plotting library has been used to create charts that are visually similar to those that are used to display certain types of bioinformatics data [JCViz]. For instance, a sequence alignment viewer can be created with a Plotly heatmap (see Fig. 15).

The Dash Bio AlignmentViewer component applies a similar approach; the React.js component uses a plotly.js heatmap to display the alignment. However, the API of AlignmentViewer differs from that of the Plotly.py heatmap. The latter requires the user to define properties of the graph that don't have anything to do with the alignment itself. Annotations must be specified, as well as a custom heatmap colorscale in which the values correspond to bases and not percentiles of a dataset. It also requires preprocessing of the FASTA data, and translation into a format that can be fit into the parameters of a Plotly heatmap.

In contrast, AlignmentViewer includes support for information that is specific to multiple sequence alignment. The gap and conservation, for instance, can be optionally included as barplots; the method of conservation can also be changed, and the consensus 
sequence can be displayed on the chart. Data in the form of FASTA files can be used as input to the component without any further processing required. This allows for the programmer to more easily interact with the component, as it removes the need to restructure data to fit a specific format for visualization.

\section{Limitations and Future Directions}

\section{File Formats}

Currently, the dash_bio_utils package only supports specific data file formats (namely, PDB, FASTA, and SOFT). Additionally, most of the components require JSON data as input; this file format is not typically provided in datasets or studies. Future developments to the package should therefore include processing for other important file formats, such as SAM/BAM/BAI for sequence alignment, or Genbank files (.gb).

\section{Conclusion}

The Dash Bio component suite facilitates visualization of common types of datasets that are collected and analyzed in the field of bioinformatics. It remains consistent with the declarative nature of Plotly's Dash, and permits users to create interactive and responsive web applications that can be integrated with other Dash components. The dash-bio-utils package additionally converts files from some of the most prominent bioinformatics databases into familiar Python data types such as dictionaries. When used in conjunction with the dash-bio package, this enables bioinformaticians to quickly and concisely communicate information among one another, and to the rest of the scientific community.

\section{REFERENCES}

[Mol3D] Autodesk. Molecule 3D for React. URL: https://github.com/plotly/ molecule-3d-for-react

[PDB] Berman, H.M., Westbrook, J., Feng, Z., Gilliland, G., Bhat, T.N., Weissig, H., Shindyalov, I.N., Bourne, P.E.. (2000) The Protein Data Bank. Nucleic Acids Research, 28: 235-242. URL:https://www.rcsb. org DOI: 10.1093/nar/28.1.235.

[Man] Bhatnagar, Samir. manhattanly. URL: https://github.com/ sahirbhatnagar/manhattanly

[cBio] Cerami, E., Gao, J., Dogrusoz, U., Gross, B. E., Sumer, S. O., Aksoy, B. A., Jacobsen, A., Byrne, C. J., Heuer, M. L., Larsson, E., Antipin, Y., Reva, B., Goldberg, A. P., Sander, C., Schultz, N..*The cBio Cancer Genomics Portal: An Open Platform for Exploring Multidimensional Cancer Genomics Data*. Cancer Discov May 1 2012 (2) (5) 401-404; DOI: 10.1158/2159-8290.CD-12-0095.

[BioP] Cock, P. J. A., Antao, T., Chang, J. T., Chapman, B. A., Cox, C. J., Dalke, A., Friedberg, I., Hamelryck, T., Kauff, F., Wilczynski, B., de Hoon, M. J. L.. Biopython: freely available Python tools for computational molecular biology and bioinformatics. Bioinformatics 25 (11), 1422-1423 (2009). DOI: 10.1093/bioinformatics/btp163.

[1bna] PDB ID: 1BNA. Drew, H.R., Wing, R.M., Takano, T., Broka, C., Tanaka, S., Itakura, K., Dickerson, R.E.. Structure of a B-DNA dodecamer: conformation and dynamics.. (1981) Proc.Natl.Acad.Sci.USA 78: 2179-2183. DOI: 10.1073/pnas.78.4.2179.

[GEO] Edgar, R., Domrachev, M., Lash, A.E.. Gene Expression Omnibus: NCBI gene expression and hybridization array data repository. Nucleic Acids Res. 2002 Jan 1;30(1):207-10. DOI: 10.1093/nar/30.1.207.

[SeqV] FlyBase. react-sequence-viewer. URL: https://github.com/FlyBase/ react-sequence-viewer

[Ghr] Genome Reference Consortium. Genome Reference Consortium Human Build 37 (GRCh37) (2009). URL: https://www.ncbi.nlm.nih. gov/assembly/GCF_000001405.13/
[cBio2] Gao, J., Aksoy, B. A., Dogrusoz, U., Dresdner, G., Gross, B., Sumer, S. O., Sun, Y., Jacobsen, A., Sinha, R. Integrative Analysis of Complex Cancer Genomics and Clinical Profiles Using the cBioPortal. Science Signaling Apr 2 2013. DOI: 10.1126/scisignal.2004088.

[Circos] Girault, N.. circosJS: d3 library to build circular graphs. URL: https: //github.com/nicgirault/circosJS

[GEOP] Gumienny, R.. GEOparse. URL: https://github.com/guma44/ GEOparse

[JCViz] johnchase. Visualizing bioinformatics data with plot.ly. URL:https: //plot.ly/ johnchase/22/visualizing-bioinformatics-data-with-plo/

[miR] Kneitz, B., Krebs, M., Kalogirou, C., Schubert, M., et al. Survival in patients with high-risk prostate cancer is predicted by miR-221, which regulates proliferation, apoptosis, and invasion of prostate cancer cells by inhibiting IRF2 and SOCS3. Cancer Res 2014 May 1;74(9):2591-603. PMID: 24607843. DOI: 10.1158/00085472.CAN-13-1606.

[KR09] Kuner, R., Muley, T., Meister, M., Ruschhaupt, M. et al. Global gene expression analysis reveals specific patterns of cell junctions in nonsmall cell lung cancer subtypes. Lung Cancer 2009 Jan;63(1):32-8. PMID: 18486272. DOI: 10.1016/j.lungcan.2008.03.033.

[NCBI] The NCBI C++ Toolkit (https://ncbi.github.io/cxx-toolkit/) by the National Center for Biotechnology Information, U.S. Fasta Sequence ID Format. National Library of Medicine; Bethesda MD, 20894 USA.

[nXP] NeXtprot. ALB - Serum albumin - proteomics. URL: https://www. nextprot.org/entry/NX_P02768/proteomics

[Dash] Plotly. Introducing Dash. (2017) URL: https://medium.com/ @ plotlygraphs/introducing-dash-5ecf7191b503

[Align] Plotly. React Alignment Viewer. URL: https://github.com/plotly/ react-alignment-viewer

[Onco] Plotly. React OncoPrint. URL: https://github.com/plotly/reactoncoprint

[Geno] Pritchard, L., White, J. A., Birch, P. R. J., Toth, I. K. GenomeDiagram: a python package for the visualization of large-scale genomic data. Bioinformatics, Volume 22, Issue 5, 1 March 2006, Pages 616-617. DOI: 10.1093/bioinformatics/btk021

[ER15] Reed, E., Nunez, S., Kulp, D., Qian, J., Reilly, M. P., and Foulkes, A. S. (2015) A guide to genome-wide association analysis and post-analytic interrogation. Statist. Med., 34: 3769- 3792. DOI: $10.1002 / \mathrm{sim} .6605$.

[Muts] Schroeder, M.. Mutations Needle Plot (muts-needle-plot). URL: https://github.com/bbglab/muts-needle-plot

[Par] Swails, J.. ParmEd. URL: https://github.com/ParmEd/ParmEd

[Speck] Terrell, R.. Speck. URL: https://github.com/wwwtyro/speck

[UniP] The UniProt Consortium. UniProt: a worldwide hub of protein knowledge. Nucleic Acids Res. 47: D506-515 (2019). DOI: 10.1093/nar/gky1049.

[Ideo] Weitz, E.. ideogram: Chromosome visualization with JavaScript URL: https://github.com/eweitz/ideogram

[PdbF] wwwPDB. Protein Data Bank Contents Guide: Atomic Coordinate Entry Format Description Version 3.30 (2008). 185-197. 